\title{
Importance of Antimicrobial Stewardship in the Treatment of Urinary Tract Infection
}

\author{
Rupinder Bakshi $^{1}$ (D), Sarjiwan Kaur $^{1}$ (D) and Vikram Jeet Singh Gill ${ }^{*}$ (D) \\ ${ }^{1}$ Department of Microbiology, Government Medical College, Patiala, Punjab, India. \\ ${ }^{2}$ All India Institute of Medical Sciences, New Delhi, India.
}

\begin{abstract}
Urinary tract infection (UTI) is a microbial contamination of the bladder and related organs. Study subjects were those who had no structural anomaly and no co-occurring diseases, such as diabetes, or were either immunocompromised or pregnant. Simple UTI is also defined as cystitis or inferior UTI. This study was a prospective, single center study conducted at a tertiary level clinic and its associated bacteriology laboratory. Patients whose urine tests were collected by the microbiology test center over a 6-month period were enrolled in the study. Culture and vulnerability results were obtained directly from the microbiology test center. Of the 1306 samples obtained, $888(68 \%)$ were from females and $418(32 \%)$ from males. This study identified the predominant UTI-causing microbes and the associated antimicrobial vulnerabilities. In males, Escherichia coli (36.8\%) was the predominant microbe followed by Klebsiella pneumoniae 19.8\%, Proteus spp. 17.4\%, Staphylococcus aureus $10.0 \%$, Pseudomonas aeruginosa $5.5 \%$, Citrobacter spp. 5.0\%, Staphylococcus saprophyticus $0.7 \%$, Enterococcus faecalis 1.4\%, and Acinetobacter spp. $0.7 \%$. In females, E. coli (49.4\%) was predominant, whereas Acinetobacter spp. $(0.3 \%)$ was the least dominant. The considerable susceptibility of $E$. coli to meropenem $(73.6 \%)$ and imipenem (73.5\%) was similar to that reported in different investigations across India. In this study, the susceptibility of $E$. coli to piperacillin + tazobactam and ciprofloxacin was $42.7 \%$ and $14.3 \%$, respectively. Stewardship of urinalysis and urine culture, especially among clinicians might be a successful upstream strategy for reducing inappropriate antimicrobial use for UTI. Thus, it is critical to routinely screen for resistance or susceptibility in samples of uropathogens, so the protocols for proper antibiotic treatment can be enhanced to incorporate antimicrobials with less resistance, supporting physicians in the appropriate treatment of UTIs resulting in insignificant remedial disappointments.
\end{abstract}

Keywords: Urinary tract infection, Uropathogens, Antimicrobial susceptibility

*Correspondence: vikramgill1@yahoo.co.in

(Received: July 05, 2021; accepted: October 04, 2021)

Citation: Bakshi R, Kaur S, Gill VJS. Importance of Antimicrobial Stewardship in the Treatment of Urinary Tract Infection. J Pure Appl Microbiol. 2021;15(4):2170-2176. doi: 10.22207/JPAM.15.4.40

(C) The Author(s) 2021. Open Access. This article is distributed under the terms of the Creative Commons Attribution 4.0 International License which permits unrestricted use, sharing, distribution, and reproduction in any medium, provided you give appropriate credit to the original author(s) and the source, provide a link to the Creative Commons license, and indicate if changes were made. 


\section{INTRODUCTION}

Urinary tract infection (UTI) is a microbial contamination of the bladder and related organs. These are subjects who have no structural anomaly and no co-occurring diseases, such as diabetes, immunocompromised, or expecting. Simple UTI is also defined as cystitis or inferior UTI. Women get affected with UTI in their lifetime, and is thus one of the most typical infections in women. ${ }^{1}$ Most simple UTIs resolve naturally without any medication; however, several women pursue treatment for indications. Treatment is meant to avoid the spread of infection to the kidneys and even as upper tract infections like pyelonephritis, which leads to damage of the sensitive tissues in the nephrons and cause hypertension. ${ }^{2}$

UTIs are a very recurrent microbial contamination in women. They commonly occur among the age groups of 16 to 35 years, with $10 \%$ of women acquiring an infection annually and more than $40 \%$ to $60 \%$ being infected at least once in their lifetime. Reoccurrences are commonly observed, with half of the women experiencing infections twice in a year. Women are affected four times more than men. ${ }^{3}$

Infective microbes travel up from the perineum and cause UTIs. Women have shorter urethras than men and hence are more liable to contract UTIs. Simple UTIs are rarely owing to blood-transmitted microbes. Escherichia coli is by far the most common microbe in simple UTIs. ${ }^{4}$ Although E. coli accounts for the maximum number of UTIs, other microbes of significance comprise Proteus, Klebsiella, and Enterococcus. The determination of UTIs is performed by obtaining the medical history of the patient and urine analysis; however, the appropriate collection of the urine sample is important. ${ }^{5}$

The main risk for UTI is the utilization of a urinary catheter. Moreover, the misuse of the urethra is also a risk. Recurrent pelvic examinations and the existence of structural anomalies of the urinary tract can also cause UTIs. A UTI is more likely to seen after a kidney transfer as both causes involve the utilization of immunosuppressors and vesicoureteral reflux. Other risk factors comprise the utilization of antimicrobials and diabetes mellitus. ${ }^{6}$ A simple UTI commonly affects the bladder. When the microbes overcome the urinary bladder mucosal wall, infection occurs. These microbes rise up to the bladder and cause a UTI. Individuals who often void and vacant the urinary bladder have less risk of UTI. ${ }^{7}$

Urine is a superlative medium for microbial development. Features that make urine ideal for microbial proliferation comprise a $\mathrm{pH}<5$, existence of organic acids, and large amounts of urea. Often, micturition is also known to reduce the danger of UTI. Microbes that lead to UTI have bonds on their surface layer which permits the microbe to get attached to the mucosal layer. Moreover, a small urethra also makes it easy for the uropathogen to infect the urinary tract. However, the use of antimicrobials can disrupt this microbial defense strategy. ${ }^{8}$

Antibiotic resistance amongst microbes leading to general infections is increasing day by day worldwide. It is noteworthy that the prevalence of resistance differs between health center and community, large and small health centers, across states, and even among nations. ${ }^{9}$ The increase in resistance to antimicrobials demonstrates the importance of using proofbased methods for infection management. In UTI incidents, antimicrobial action is generally initiated analytically before the results of urine culture and vulnerability testing are available.

Antibiotic resistance among microorganisms causing normal diseases is expanding worldwide. The development of resistance to antimicrobials emphasizes the importance of utilizing proof-based methodologies for infection management. In UTI incidents, antibiotic treatment is frequently begun before the results of urine culture and vulnerability tests are available. Following of proper anti-infection protocols in patients with a UTI appears to decrease the span of clinic stay, and subsequently, favors patient outcomes and reduces medical expenditure. ${ }^{10}$

Considering the importance of each of the described factors, this study aimed to examine and identify the causative agents of UTI and their patterns of antibiotic susceptibility. Such an evaluation can help design a proof-based medication strategy and aid in monitoring of antibiotic susceptibility patterns.

\section{MATERIAL AND METHODS}

This was a review, expressive, single 
center study conducted at a tertiary level clinic and its associated bacteriology laboratory. This clinic addresses the food needs of patients from areas close to the metropolitan center and country regions and those of patients referred from different areas. Patients whose urine tests were collected by the microbiology test center over a 6-month period were enrolled in this study. Culture and sensitivity test results were obtained directly from the microbiology test center.

\section{Sample collection and processing}

Hygienic mid-stream catch urine samples, those acquired by draining from a catheter, or by suprapubic suction were collected in clean, extensive mouthed standard vessels. Patients with entirely assumed UTI (outside/nomadic subjects and inside/hospitalized subjects) were selected and handled by the microbiology test center. Contaminated samples were disposed off and not handled.

\section{Culture and identification of isolates}

Urine tests were cultured on suitable culture media by utilizing semi quantifiable techniques and culture media was maintained for 2 days at $37{ }^{\circ} \mathrm{C}$. Cultures were first analyzed for development and area coverage indicated the presence of considerable or unimportant bacteriuria. The development of $\geq 10^{5}$ colony

\section{Percentage of patients according to age group}

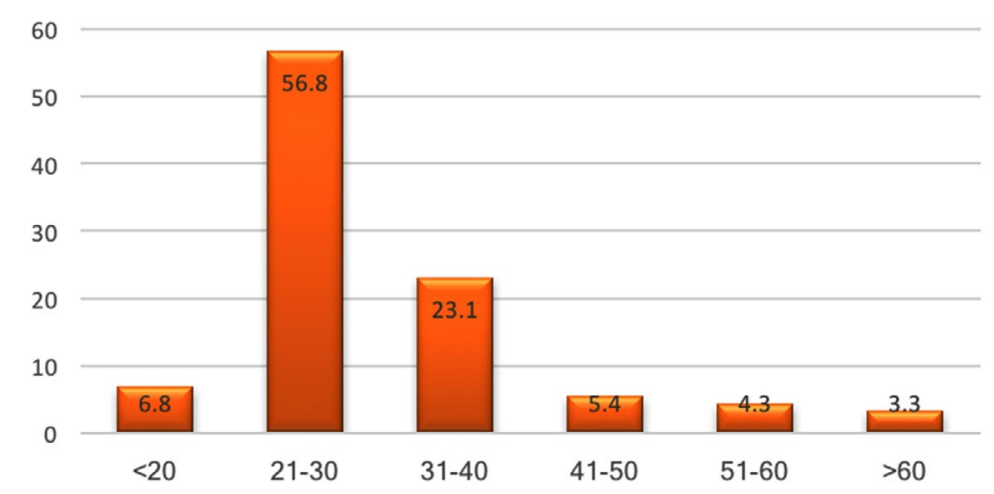

Fig. 1. Distribution of the Percentage of patients according to age group.

\section{GENDER WISE DISTRIBUTION}

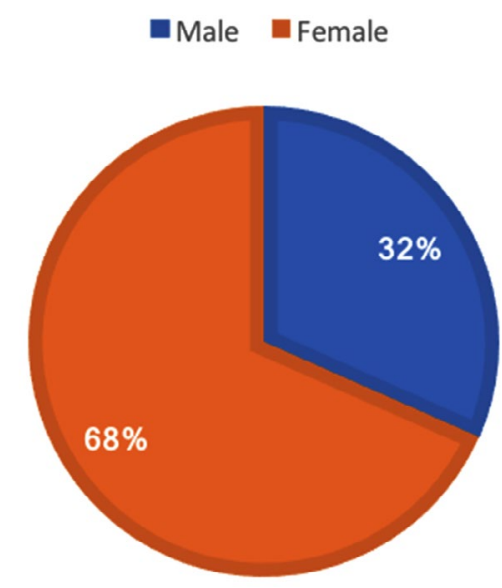

Fig. 2. Gender wise distribution. forming units/mL was considered as critical bacteriuria, indicative of UTI. Identification of cultures was performed based on previously described biochemical and other laboratory investigations. $^{11}$

\section{Antimicrobial susceptibility pattern}

Antimicrobial vulnerability assessments were performed using the Improved Kirby Bauer Disc Diffusion technique on Muller Hinton Agar as per the CLSI values. ${ }^{12}$ The antimicrobials tested were ampicillin $(10 \mu \mathrm{g})$, amikacin (30 $\mu \mathrm{g})$, gentamicin $(10 \mu \mathrm{g})$, ciprofloxacin $(5 \mu \mathrm{g})$, levofloxacin $(5 \mu \mathrm{g})$, ofloxacin $(5 \mu \mathrm{g})$, norfloxacin $(10 \mu \mathrm{g})$, ceftazidime $(30 \mu \mathrm{g})$, cefotaxime $(30 \mu \mathrm{g})$, ceftriaxone $(30 \mu \mathrm{g})$, cefepime $(30 \mu \mathrm{g})$, piperacillintazobactum $(100 / 10 \mu \mathrm{g})$, nitrofurantoin (300 $\mu \mathrm{g})$, cotrimoxazole $(25 \mu \mathrm{g})$, imipenem $(10 \mu \mathrm{g})$, meropenem $(10 \mu \mathrm{g})$, and aztreonam $(30 \mu \mathrm{g})$. 
Table 1. Gender based categorisation of several uropathogens in culture positive specimens

\begin{tabular}{lccc}
\hline Name of the organism & Male, $\mathrm{n}(\%)$ & Female, $\mathrm{n}(\%)$ & Overall, $\mathrm{n}(\%)$ \\
\hline Escherichia coli & $154(36.8)$ & $492(55.4)$ & $646(49.4)$ \\
Klebsiella pneumoniae & $83(19.8)$ & $212(23.8)$ & $295(22.5)$ \\
Pseudomonas aeruginosa & $23(5.5)$ & $18(2.0)$ & $41(3.1)$ \\
Acinetobacter spp. & $3(0.7)$ & $1(0.1)$ & $4(0.3)$ \\
Enterococcus faecalis & $6(1.4)$ & $2(0.2)$ & $8(0.6)$ \\
Proteus spp. & $73(17.4)$ & $71(7.9)$ & $144(11.0)$ \\
Staphylococcus aureus & $42(10.0)$ & $63(7.0)$ & $105(8.0)$ \\
Staphylococcus saprophyticus & $13(3.1)$ & $5(0.5)$ & $18(1.3)$ \\
Citrobacter spp. & $21(5.0)$ & $24(2.7)$ & $45(3.4)$ \\
Total & $418(32.0)$ & $888(68.0 \%)$ & $1306(100)$ \\
\hline
\end{tabular}

Table 2. Antimicrobial susceptibility pattern of isolated organisms

\begin{tabular}{lcc}
\hline $\begin{array}{l}\text { Antibiotic } \\
\text { drugs }\end{array}$ & $\begin{array}{c}\text { Escherichia coli } \\
646(\%)\end{array}$ & $\begin{array}{c}\text { Klebsiella spp. } \\
295(\%)\end{array}$ \\
\hline Amikacin & $321(49.6)$ & $134(45.4)$ \\
Ampicillin & $143(22.1)$ & $19(6.4)$ \\
Amoxy-clav & $258(39.9)$ & $89(30.1)$ \\
Aztreonam & $374(57.8)$ & $239(81.0)$ \\
Cefotaxime & $143(22.1)$ & $47(15.9)$ \\
Ceftrioxane & $141(21.8)$ & $36(12.2)$ \\
Cefuroxime & $97(15.0)$ & $54(18.3)$ \\
Cefatzidime & $321(49.6)$ & $153(51.8)$ \\
Ciprofloxacin & $93(14.3)$ & $73(24.7)$ \\
Cotrimoxazole & $173(26.7)$ & $81(27.4)$ \\
Gentamicin & $271(41.9)$ & $103(34.9)$ \\
Imipenem & $475(73.5)$ & $153(51.8)$ \\
Levofloxacin & $123(19.0)$ & $79(26.7)$ \\
Meropenem & $476(73.6)$ & $173(58.6)$ \\
Nitrofurantoin & $374(57.8)$ & $115(38.9)$ \\
Norfloxacin & $183(28.3)$ & $72(24.4)$ \\
Ofloxacin & $321(49.6)$ & $89(30.1)$ \\
Piperacillin + & $276(42.7)$ & $73(24.7)$ \\
tazobactam & & \\
\hline
\end{tabular}

Antibiotics included for gram-positive cocci were ampicillin $(10 \mu \mathrm{g})$, amoxycillin $(30 \mu \mathrm{g})$, amoxy-clav (20/10 $\mu \mathrm{g})$.

\section{RESULT}

A total of 1306 patients who satisfied the inclusion principles during the investigation were enlisted. The information was examined, and the last perceptions were classified as described. In this study, the maximum number of subjects $(56.8 \%, n=743)$ were in the age group of $21-30$ years followed by those in the age group 31-40 years $(23.1 \%, n=302)$ as shown in Fig. 1 .

In Fig. 2, of the 1306 samples, 888 were from females and 418 from males, which correspond to $68 \%$ female and $32 \%$ male in Table 2.

Table 1 presents the UTI-causing pathogens identified in this study and their levels of antimicrobial vulnerability. In males, E. coli (36.8\%) was the chief pathogen followed by Klebsiella pneumoniae (19.8\%), Proteus spp. (17.4\%), Staphylococcus aureus (10.0\%), Pseudomonas aeruginosa (5.5\%), Citrobacter spp. (5.0\%), Staphylococcus saprophyticus (0.7\%), Enterococcus faecalis (1.4\%), and Acinetobacter spp. (0.7\%). In females, E. coli $(49.4 \%)$ was the most predominant and Acinetobacter spp. (0.3\%) the least.

As shown in Table 2, E. coli was the most susceptible to meropenem (73.6\%) and imipenem $(73.5 \%)$, which was consistent with the findings in other studies in India. In this study, vulnerability of E. coli to piperacillin + tazobactam was 42.7 and that of $E$. coli to ciprofloxacin was $14.3 \%$.

\section{DISCUSSION}

Treatment of a UTI is best overseen in an interdisciplinary style; moreover, almost all clinicians have experienced a patient having a UTI. The strategy to avoiding repeated infections is to raise awareness of the patient. When a UTI is diagnosed, the patient should be urged to consume more liquids. Some women with intermittent UTIs may benefit with the prophylactic use of anti-infection agents. Other non-clinical cures 
may assist other women with UTI. Importantly, clinicians should refer patients with repetitive UTIs to a urologist to preclude reflux and structural imperfections. The earlier a UTI is dealt with, the better are the results, and inclusion of an interdisciplinary group is critical for improved results.

In our study, the results obtained show that the range of organic entities caused UTI is similar to that reported in different studies across India. However, isolation levels of different microorganisms differed from study to study. ${ }^{9}$ In our study, women were the majority, and isolation rates between samples of men and women were quite distinct, which was similar to the several studies led across India, in which the isolation rates were discovered to be more in women. ${ }^{10}$ To a limited extent, this disparity could be a direct result of the more number of serious UTIs in women thereby resulting in the increased number of requests for culture and vulnerability assessments and considering that infection management of most simple UTIs is generally conducted observationally without resorting to culture and vulnerability assessments.

In our study, E. coli (49.4\%) was the well-known disengaged life form responsible for causing UTIs in our samples, consistent with results of different studies across India. ${ }^{11}$ A high $E$. coli confinement level of $69.8 \%$ was found in the study by Keren et al. ${ }^{12}$ with increased susceptibility of $E$. coli to meropenem (73.6\%) and imipenem (73.5\%) similar to that observed in different investigations across India. However, the study by Ahmed et al. ${ }^{13}$ revealed a low level of $E$. coli susceptibility $(39.5 \%)$ to imipenem. Susceptibility of $E$. coli to nitrofurantoin was found to be high at $57.8 \%$, consistent with reports from different studies across India. ${ }^{14}$ In this study, the susceptibility of $E$. coli to piperacillin + tazobactam was found to be $42.7 \%$, whereas it ranged from $15.15 \%$ to $52.3 \%$ in different studies across India. ${ }^{15}$ Vulnerability of E. coli to ciprofloxacin was $14.3 \%$ which was lower than that of the susceptibility rates observed in different studies across India. ${ }^{16}$ High susceptibility levels of $E$. coli to ciprofloxacin was reported in studies conducted in Poland (65.8\%) and Ethiopia $(54.8 \%)$ showing geographical variations in antibiotic susceptibility patterns. ${ }^{17,18}$
In our study, Klebsiella pneumoniae was the second most isolated uropathogen at a level of $22.5 \%$, similar to the isolation level of $18.71 \%$ reported in study from North India. ${ }^{19}$ Ina ddition, Klebsiella pneumoniae was the second most regularly isolated microbe in different studies across India. ${ }^{20}$ Susceptibility to nitrofurantoin, cotrimoxazole, and ciprofloxacin was found to be $38.9 \%, 27.4 \%$, and $24.7 \%$, respectively. Susceptibility of Klebsiella isolates to nitrofurantoin ranged from $38 \%$ to $67 \%$ in studies across India. ${ }^{21}$

Protection from antimicrobials is more in India in contrast to that in countries such as the UK, USA, Australia, and South Africa. ${ }^{23}$ Reduced Susceptibility of uropathogens to empiric antimicrobials prescribed for UTI such as cotrimoxazole and ciprofloxacin and even to a wide range of anti-toxins in India, as clearly indicated by this and previous studies, could be a direct result of the extensive utilization of antiinfection agents owing to a number of factors. The tendency for self-medication, refusal of treatment, monetary requirements, absence of instruction on piece of patient, offer of antiinfection drugs deprived of legitimate medicine and inability drug specialists to provide adequate information to patients, irrelevant reconnaissance of susceptibility designs, poor administrative regulations regarding antimicrobials, absence of will to make change on piece of medical care framework, and administration of anti-infection agents before obtaining culture tests, and poor endorsing rehearses by doctors are amongst the numerous elements that lead to the rash and irresponsible utilization of antitoxins in India, consequently causing rapid advancement of resistance. ${ }^{24}$

Based on the results of this study, it is suggested that nitrofurantoin, rather than cotrimoxazole and ciprofloxacin, is preferable for use as an empiric antitoxin for simple cystitis. For simple and complex pyelonephritis, aminoglycosides and carbapenems ought to be preferred over fluoroquinolones and cephalosporins. In all cases, testing of urine for culture and susceptibility testing ought to be performed before selection of the anti-toxins and later treatment ought to be adjusted to 
a narrow range of agents according to urine culture and susceptibility result. This study emphasizes the need for emergency clinic or territorial antibiograms to battle the issue of antibiotic confrontation. Antibiograms help screen antimicrobial resistance patterns and aid physicians in the selection of the appropriate antibiotic treatment.

Antimicrobial resistance systems can be categorized into four fundamental classes: (1) restricting take-up of a medication, (2) altering a medication target, (3) inactivating a medication, and (4) dynamic medication efflux. Natural resistance mechanisms may utilize restricting take-up, drug inactivation, and medication efflux; procured resistance mechanism utilized might be drug target change, drug inactivation, and medication efflux. Owing to the diversity in resistance patterns, there is variety in the kinds of systems utilized by gram-negative microorganisms versus gram-positive mciroorganisms. Gramnegative microorganisms utilize each of the four principles components, whereas gram-positive microorganisms less regularly utilize restricting the take-up of a medication (as they do not possess a lipopolysaccharide external film), and do not have a limit with respect to the specific types of medication efflux systems.

\section{CONCLUSION}

Stewardship of urinalysis and urine culture, especially among clinicians, might be a successful upstream strategy for reducing the improper use of antimicrobials for UTI. Therefore, it is critical to routinely screen for resistance or susceptibility in samples examples of uropathogens, so the protocols for proper antibiotic treatment can be enhanced to incorporate antimicrobials with low resistance, supporting clinicians in the legitimate administration of UTIs with insignificant remedial disappointments. Clinicians should work closely with a microbiologist to guarantee the best antimicrobial decisions for treatment. Patient and local area security are influenced by guaranteeing the prescribing of the best antimicrobial and medicine consistently.

\section{ACKNOWLEDGMENTS}

None.

\section{CONFLICT OF INTEREST}

The authors declare that there is no conflict of interest.

\section{AUTHORS' CONTRIBUTION}

All authors listed have made a substantial, direct and intellectual contribution to the work, and approved it for publication.

\section{FUNDING}

None.

\section{DATA AVAILABILITY}

All datasets generated or analyzed during this study are included in the manuscript.

\section{ETHICS STATEMENT}

Not applicable.

\section{REFERENCES}

1. Wagenlehner F, Wullt, B, BallariniS. Social and economic burden of recurrent urinary tract infections and quality of life: a patient web-based study (GESPRIT). Expert Rev Pharmacoecon Outcomes Res. 2018;18(1):107-117. doi: 10.1080/14737167.2017.1359543

2. Uwaezuoke SN. The prevalence of urinary tract infection in children with severe acute malnutrition: a narrative review. Pediatric Health Med Ther. 2016;7:121-127. doi: 10.2147/PHMT.S107421

3. Abelson B, Sun D, Que L, et al. Sex differences in lower urinary tract biology and physiology. Biol Sex Differ. 2018;9(1):45. doi: 10.1186/s13293-018-0204-8

4. Sutton AG, Chandler N, Roberts KB. Recent Studies on the Care of First Febrile Urinary Tract Infection in Infants and Children for the Pediatric Hospitalist. Rev Recent Clin Trials. 2017;12(4):269-276. doi: 10.2174/ 1574887112666170816143639

5. Swerkersson S, Jodal U, Sixt R, Stokland E, Hansson S. Urinary tract infection in small children: the evolution of renal damage over time. Pediatr Nephrol. 2017;32(10):1907-1913. doi: 10.1007/s00467-0173705-5

6. Wu CT, Lee $\mathrm{HY}$, Chen $\mathrm{CL}$, Tuan $\mathrm{PL}$, Chiu $\mathrm{CH}$. High prevalence and antimicrobial resistance of urinary tract infection isolates in febrile young children without localizing signs in Taiwan. J Microbiol Immunol Infect. 2016;49(2):243-248. doi: 10.1016/j. jmii.2015.05.016

7. Tandogdu Z, Wagenlehner FM. Global epidemiology of urinary tract infections. Curr Opin Infect Dis. 2016;29(1):73-79. doi: 10.1097/ QCO.0000000000000228

8. Kranz J, Schmidt S, Lebert C. The 2017 update of the German clinical guideline on epidemiology, diagnostics, therapy, prevention, and management of uncomplicated urinary tract infections in adult patients: part 1. Urol Int. 2018;100(3):263-270. doi: 


\section{$10.1159 / 000487645$}

9. Stein R, Dogan HS, Hoebeke $P$, et al. Urinary tract infections in children: EAU/ESPU guidelines. Eur Urol. 2015;67(3):546-558. doi: 10.1016/j. eururo.2014.11.007

10. Kant S, Lohiya A, Kapil A, Gupta SK. Urinary tract infection among pregnant women at a secondary level hospital in Northern India. Indian J Public Health. 2017;61:118-123. doi: 10.4103/ijph.IJPH_293_15

11. Sharma N, Gupta A, Walia G, Bakhshi R. Pattern of antimicrobial resistance of Escherichia coli isolates from urinary tract infection patients: A three year retrospective study. J Appl Pharm Sci. 2016;6(1):62-65. doi: 10.7324/JAPS.2016.600110

12. Keren R, Shaikh N, Pohl H, et al. Risk Factors for Recurrent Urinary Tract Infection and Renal Scarring. Pediatrics. 2015;136(1):e13-21. doi: 10.1542/ peds.2015-0409

13. Ahmed M, Moremi N, Mirambo MM, et al. Multiresistance gram-negative enteric bacteria causing urinary tract infection among malnourished underfives admitted at a tertiary hospital, northwestern, Tanzania. Ital J Pediatr. 2015;41:44. doi: 10.1186/ s13052-015-0151-5

14. Laxminarayan R, Chaudhury RR. Antibiotic resistance in India: Drivers and opportunities for action. PLOS Med. 2016;13(3):e1001974. doi: 10.1371/journal. pmed.1001974

15. Kulkarni SR, Peerapur BV, Sailesh KS. Isolation and antibiotic susceptibility pattern of Escherichia coli from urinary tract infections in a tertiary care hospital of North Eastern Karnataka. J Nat Sci Biol Med. 2017;8(2):176-180. doi: 10.4103/0976-9668.210012

16. Patel HB, Soni ST, Bhagyalaxmi A, Patel NM Causative agents of urinary tract infections and their antimicrobial susceptibility patterns at a referral center in Western India: An audit to help clinicians prevent antibiotic misuse. J Family Med Prim Care. 2019;8(1):154-159. doi: 10.4103/jfmpc.jfmpc_203_18
17. Stefaniuk E, Suchocka U, Bosacka K, Hryniewicz W. Etiology and antibiotic susceptibility of bacterial pathogens responsible for community acquired urinary tract infections in Poland. Eur J Clin Microbiol Infect Dis. 2016;35(8):1363-1369 doi: 10.1007/s10096-016-26731

18. Mamuye $Y$. Antibiotic resistance patterns of common gram negative uropathogens in St. Paul's hospital millennium medical college. Ethiop J Health Sci. 2016;26(2):93-100. doi: 10.4314/ejhs.v26i2.2

19. Khan R, Saif Q, Fatima K, Meher R, Shahzad HF, Anwar KS. Clinical and bacteriological profile of UTI patients attending a North Indian tertiary care center. J Integr Nephrol Androl. 2015;2(1):29-34. doi: 10.4103/22251243.150009

20. Pardeshi P. Prevalence of urinary tract infections and current scenario of antibiotic susceptibility pattern of bacteria causing UTI. Indian I Microbiol Res. 2018;5(3):334-338. doi: 10.18231/23945478.2018 .0070

21. Singh RK, Dewasy B, Mallick RL, Kafle TK. Prevalence of antibiotic sensitivity pattern of uropathogens in patients of different age-groups from western region of Nepal. Int J Med Sci Public Health. 2016;5(9):1-7.

22. Ansari MS, Shekar PA, Singh C, Joshi SS. The Urological Society of India Guidelines for the management of pediatric urinary tract infection. Indian J Urol. 2021;37:10-12. doi: 10.4103/iju.IJU_568_20

23. Centre for Disease Dynamics Economics and Policy. Resistance Map. Washington DC, USA: Center for Disease Dynamics, Economics and Policy; 2017. https:// www.resistancemap.cddep.org/AntibioticResistance. php. Accessed on 2018 Aug 01.

24. Yashavanth R, Shiju MP, Bhaskar UA, Ronald R, Anita KB. Candiduria: Prevalence and trends in antifungal susceptibility in a tertiary care hospital of Mangalore. J Clin Diagn Res. 2013;7(11):2459-2461. doi: 10.7860/ JCDR/2013/6298.3578 